\title{
RATURER LA THÉORIE MIMÉTIQUE : MARION AU-DELÀ DE GIRARD
}

\author{
Stéphane Vinolo* \\ doi: 10.11144/Javeriana.uph35-71.rtmm
}

\section{RÉSUMÉ}

La théorie mimétique de René Girard est totalement close sur la violence parce que la déconstruction des mécanismes victimaires, mis à nu sur la Croix, n'a fait qu'accélérer l'advenue de l'Apocalypse. En montrant que cette clôture est due à un enfermement métaphysique d'un premier moment de la théorie mimétique, enfermement porté par un chemin qui nous mène inexorablement de l'objet à l'être, en passant par l'image et l'idole, nous ouvrons une brèche dans la violence grâce à la phénoménologie de la donation de Marion. Contre le modèle de l'image et de son idolâtrie, Marion nous permet de dépasser la violence par le paradigme de l'icône qui, loin d'en passer par l'image et l'être, nous porte vers le signe et les phénomènes saturés. En percevant le monde non plus à l'aune de l'objectité, mais selon la modalité phénoménologique de la saturation, la boucle autoréférentielle de la violence mimétique est mise en question et nous ouvre la possibilité de relations pacifiques. Aussi, le grand défi de notre temps n'est pas tellement de savoir donner que d'apprendre à recevoir.

Mots clés: Girard ; icône ; idole ; Marion ; phénomènes saturés

Pontificia Universidad Católica de Ecuador.

Correo electrónico: svinolo@puce.edu.ec

Para citar este artículo: Vinolo, S. (2018). Raturer la théorie mimétique : Marion au-delà de Girard. Universitas Philosophica, 35(71), pp. 201-231. ISSN 0120-5323, ISSN en línea 2346-2426.

doi: 10.11144/Javeriana.uph35-71.rtmm 


\title{
CROSSING OUT MIMETIC THEORY: MARION BEYOND GIRARD
}

\begin{abstract}
The mimetic theory forged by René Girard is completely closed upon its own violence. Given that human violence was contained for centuries, by the victimary mechanism, its deconstruction by the Cross has unleashed a complete and total violence, leading us to the possibility of a real Apocalypse. We show here that this closure is based on a metaphysical view of desire, guiding us from the objet to the being of the model. Since Girard is always confined into metaphysics by his constant use of the paradigm of the image, the presence and, consequently, the idol, we demonstrate that there is still a way out of violence, the same way that is today the way out of metaphysics. With Jean-Luc Marion and his phenomenology of givenness, we oppose the paradigm of the icon to the one of the idol, and therefore the model of the sign to the one of the image. We demonstrate then that global mimetic violence can be stopped if we no longer desire objects and start desiring saturated phenomena, which implies a complete shift in the way we think and receive phenomena.
\end{abstract}

Keywords: Girard; icon; idol; Marion; saturated phenomena 
Nous connaissons aujourd'hui, après la violence des armes (la guerre) et la violence des mots (l'idéologie), la terreur de l'image idolâtrique; comme les précédentes, cette violence en veut à nos âmes - mais, pour y parvenir, elle n'exerce plus de chantage sur nos corps (comme la guerre), ou notre intelligence (comme l'idéologie). Elle s'empare de notre désir lui-même : la tyrannie de l'image idolâtrique nous vainc avec notre consentement empressé. Jean- Luc Marion, La croisée du visible, p. $98 .{ }^{1}$

Nous POURRIONS PENSER que jusqu'à la parution d'Achever Clausewitz en 2007, et même à l'intérieur de ce texte, la pensée de Girard s'orientait de façon inexorable vers un pessimisme de plus en plus sombre, nous ouvrant la seule Apocalypse comme horizon indépassable de l'humanité. Comme ne cesse de le répéter Girard, nous savons maintenant que les chemins que nous empruntons ne nous mènent nulle part et qu'il n'y aura pas de marche arrière sur le chemin de la violence : « Nous pouvons tous participer à la divinité du Christ, à condition de renoncer à notre violence. Nous savons maintenant, en partie grâce à Clausewitz, que les hommes n'y renonceront pas. » (Girard, 2007, p. 20). Paradoxalement, le chemin montré par le Christ a non seulement échoué à mettre à mal la violence, mais il a de plus fait tomber le seul rempart efficace dont nous disposions afin de nous en protéger, nous exposant désormais pleinement à son déchaînement infernal : « le verrou du meurtre fondateur, levé par la Passion, libère aujourd'hui une violence planétaire, sans qu’on puisse refermer ce qui a été ouvert. » (Girard, 2007, p. 12). La Révélation, en déconstruisant le processus qui nous permettait, depuis l'origine de la Culture, de maintenir la paix entre les Hommes - la logique du bouc émissaire et de l'expulsion de Satan par Satan -, n’a fait qu'aggraver le degré de violence auquel il nous faut maintenant faire face ${ }^{2}$. Puisqu'il n'y aura

1 Sauf indication contraire, c'est toujours nous qui soulignons.

2 Nous avons proposé ailleurs, un modèle politique pouvant se poser comme nouveau rempart à la violence, mais il nous a alors fallu montrer comment celui-ci devait relever d'une politique infondée. Voir Vinolo, 2013. 
pas de conversion soudaine et globale, nous voilà condamnés à l'Apocalypse par le geste même de celui qui a pourtant exposé, dans leur nudité la plus crue, sur la Croix, les règles structurelles de la violence. Le système girardien semble donc clos par sa propre mise au jour des mécanismes qui fondent la violence.

Un fait doit toutefois nous permettre de mettre en doute cette fermeture, et nous pousse à réinterroger la théorie mimétique dans son ensemble depuis son cœur : le désir mimétique et son objet. Certes, les études sur le désir mimétique ne manquent pas, et l'œuvre de Girard a enfin trouvé sa juste place dans le monde des idées au niveau international. On a pu ainsi questionner a un niveau fondamental le caractère mimétique du désir, ou la plus ou moins grande proximité du médiateur ainsi que l'opportunité de la différence entre médiateur interne et médiateur externe ; on a encore interrogé la forme singulière ou plurielle du médiateur du désir, ou encore le caractère inévitable du lien entre désir mimétique et violence. Pourtant, malgré les nombreux efforts des commentateurs, les voies semblent définitivement fermées. Faute de conversion globale, la violence et l'Apocalypse menacent de façon inévitable l'humanité. Nous souhaitons questionner ici cette clôture en plongeant au cœur de la théorie du désir mimétique, et en travaillant non pas sur le caractère mimétique du désir, ni sur les distances des médiateurs, pas même sur la création du désirable par le désiré, mais en mettant au cœur de la réflexion le statut objectal de ce qui est désiré ainsi que le cheminement inévitable du désir de l'objet vers l'être. Ainsi, ce n'est pas tant la conception proprement mimétique du désir qui nous occupe ici que celle du désir en tant que tel. Certes, dans La violence et le sacré, Girard a reproché à Freud de s'en être tenu à une conception objectale du désir contre une intuition qui aurait dû l'amener à penser son caractère mimétique ${ }^{3}$, mais plus que l'objectalité du désir au sens freudien, c'est son objectité phénoménologique que nous souhaitons questionner. Nous souhaitons donc montrer que la clôture sur la violence

3 Pour Girard, Freud a bien pensé un désir de l'enfant provenant de l'identification au père, et donc un désir très proche du désir mimétique. Mais Freud a malheureusement rajouté à ce désir un second désir - désir de la mère - qui est autonome de toute identification au père, et se construit donc comme désir objectal, reliant directement un sujet et un objet, sans passer par un quelconque médiateur (Girard, 1972, pp. 502-529). 
du premier moment de la théorie mimétique repose sur une conception du désir qui ne le pense quà l'aune du désir d'objet ${ }^{4}$.

C’est avec la phénoménologie de la donation de Jean-Luc Marion que nous nous proposons de faire. Certes, cette phénoménologie et la théorie mimétique ne semblent pas liées l'une à l'autre. Leurs sources sont différentes (grande tradition philosophique pour l'une contre la littérature et l'anthropologie pour l'autre) et leurs problématiques le sont aussi (description des phénomènes saturés en intuition pour l'une, mise au jour de la mécanique du désir pour l'autre). Toutefois, nous pouvons les mettre en relation par le concept d'objet. Girard pense la violence mimétique à partir de la construction des objets désirables par la focalisation sur ceux-ci de multiples désirs. L’objet qui apparaît comme désirable est avant tout l'objet qui est désiré. Même si le désir n'est pas objectal mais mimétique, c'est d'un désir d'objet que part Girard avant que d'en passer au désir métaphysique de l'être du médiateur. De l'autre côté, Marion ouvre la phénoménologie au-delà de l'objectité parce quelle fait obstacle à la donation radicale en lui fixant des limites subjectives et donc en la conditionnant (Vinolo, 2012). Dans les deux cas donc, les auteurs partent des objets dans le but de les dépasser. Mais alors que le premier Girard en arrive à un système clos sur sa propre violence, Marion rouvre la philosophie par une phénoménologie non-objectale. Nous pouvons donc, avec Marion, nous demander ce qu'il en est de la théorie mimétique sitôt que nous acceptons de dépasser les frontières de l'objectité afin de voir si la clôture radicale du système se maintient, ou si celle-ci ne provenait que de l'objectité première du désiré. C'est là ce que nous appelons, suivant en cela Marion, la rature, qui retrouve son sens positif de dépassement, de marque de l'absence au cœur même de la présence. En raturant la théorie mimétique, nous ne souhaitons donc pas la mettre à mal, encore moins la réfuter, mais mettre au jour les fondements philosophiques du tournant levinassien de René Girard ${ }^{5}$, en

4 Ce point explique probablement le tournant levinassien du dernier Girard, obligé de réintroduire du non-objectal dans les relations inter-individuelles.

5 Il est tout à fait notable que Girard et Marion se tournent vers Levinas. Encore que les relations entre Marion et Levinas soient plus complexes (Vinolo, 2016) que celles entre Levinas et Girard, nous souhaitons montrer que c'est dans les deux cas un problème d'objéctité et d'altérité qui les réunit. 
montrant pourquoi Girard s'est trouvé face à la nécessité - tout comme Levinas (Levinas, 1961) et Marion - de dépasser la métaphysique de la présence et de l'objet.

\section{Clôtures girardiennes}

LE COEUR DE LA THÉORIE MIMÉTIQUE est le caractère triangulaire du désir. Mais ce n'est pas tellement celui-ci qui pose problème. La difficulté est qu'au cœur même du triangle, nous ne pouvons pas distinguer de façon claire et distincte le modèle de celui qui l'imite ou de celui qu'il imite. Chacun étant à la fois modèle et imitateur, la structure statique du triangle se transforme en spirale dynamique dans laquelle chacun occupe de façon successive, et même simultanée, chacun des deux rôles. Ce faisant, la violence provient du fait que chacun devient à la fois modèle mais aussi rival de l'autre. C'est là le moment de la « médiation double. ${ }^{6}$ Néanmoins, puisque ce triangle mimétique, avant même qu'il ne devienne spirale, est bien formé de trois sommets (sujet - objet - médiateur), nous pourrions imaginer jouer sur chacun d'entre eux afin d'éviter que la spirale mimétique, en transformant les modèles-imitateurs en rivaux, ne sombre dans la spirale de la violence. Il y aurait ainsi trois solutions structurelles possibles à l'Apocalypse, une pour chacun des pôles du triangle. Néanmoins, les trois sont un échec, d'où la clôture structurelle du système girardien et notre volonté de la questionner à partir de l'objectité afin de montrer la trace originaire de la nécessité de l'ouvrir par l'altérité levinassienne.

Afin de ne pas sombrer dans la violence, nous pourrions d'abord jouer sur la démultiplication des objets. Dans le triangle, il y aurait une première solution qui frapperait le pôle « objet ». Puisque le fait de ne pas pouvoir partager un objet provoque la violence entre les sujets le désirant, en faisant de tous des rivaux, nous pouvons imaginer que rendre partageables les objets apporte une solution au problème. C'est là en un sens le pari de l'économie de marché qui transforme les objets en marchandises et les démultiplie, mettant à mal la violence en supprimant le problème de la rareté. Si tout le monde désire un même objet, mais

6 « Nous avons maintenant un sujet-médiateur et un médiateur-objet, un modèle-disciple et un disciple-modèle. » (Girard, 1961, p. 115). 
que cet objet, produit à la chaîne, peut être démultiplié à l'infini, chacun devrait logiquement pouvoir en jouir et satisfaire son désir de possession sans entrer en concurrence avec qui que ce soit. Malheureusement, c'est là oublier le caractère proprement mimétique du désir et sous-estimer le fait que ce que nous montre la théorie girardienne est que bien plus que de posséder l'objet, nous souhaitons en priver l'autre : « Dans la médiation double, on ne désire pas tant l'objet qu'on ne redoute de le voir posséder par autrui. » (Girard, 1961, p. 117). La démultiplication des objets en marchandises ne met pas fin à la violence parce que l'économie confond, au sein de la mêmeté, l'ipse et l'idem. En effet, « même » peut se rendre en latin par ipse ou par idem, mais c'est du côté de l'idem que se situe la théorie mimétique, et la différence est de taille. La mêmeté au sens de l'idem a ceci de particulier qu'au cœur du « même » elle implique l'altérité. Pour que cette voiture soit la même que telle autre - au sens de idem -, elle doit aussi être « autre », sans quoi elle ne serait pas « la même que » mais la voiture « elle-même ». Or, lorsque la théorie mimétique montre pourquoi et comment les hommes désirent les mêmes objets, c'est bien d'une mêmeté au sens de ipse dont il s'agit. Plus que de désirer le même objet (idem) que celui que désire le médiateur, nous désirons cet objet lui-même (ipse). Donc, l'économie de marché se trompe en répondant au problème de la violence produite par la mêmeté des objets désirés en interprétant cette mêmeté selon l'idem, alors qu'il s'agit d'une mêmeté au sens de ipse. L'économie de marché aurait d'ailleurs dû savoir qu'elle mésinterprétait cette mêmeté par le simple fait qu'elle-même cherche toujours à redifférencier les objets produits à la chaîne, comme nous le voyons dans son obsession des « séries limitées ». La simple existence de ces « séries limitées » est bien la marque que le désir mimétique ne se contente pas de vouloir posséder les mêmes objets que tout le monde, mais des objets différenciants ${ }^{7}$. La démultiplication des objets ne suffit donc pas à faire obstacle à la violence mimétique parce que nous ne désirons pas les mêmes objets au sens de idem mais l'objet lui-même (au sens de ipse), celui-là même que les autres désirent. C'est d'ailleurs ce que perçoit parfaitement Girard dès ses analyses de 1961 : «Les autres objets n'ont aucune valeur aux yeux de l'envieux, seraient-ils analogues ou même

7 Nous avons déjà développé ailleurs le concept de « contre-productivité rationnelle de la différence » à propos d'un rapprochement entre Girard et Sartre (Vinolo, 2010). 
identiques à l'objet "médiatisé". » (Girard, 1961, p. 45). La lutte contre la violence se focalisant sur le pôle « objet » du triangle est donc un échec parce que la mêmeté, telle que la pose la théorie du désir mimétique, est une identité close, d'où sa violence inévitable.

Nous pourrions imaginer alors de lutter contre la violence mimétique en travaillant sur le pôle «médiateur » du triangle. Puisque la relation de médiation interne porte la violence, il suffirait de toujours nous en tenir à des médiateurs externes, médiateurs avec lesquels nous ne pouvons jamais entrer en concurrence et qui demeurent de simples médiateurs sans ne jamais devenir des rivaux. Ce choix des médiateurs est d'ailleurs une alternative que nous retrouvons dans le texte de Girard : « Choisir, c'est toujours se choisir un modèle et la liberté véritable se situe dans l'alternative fondamentale entre modèle humain et modèle divin. » (Girard, 1961, p. 80). Une focalisation sur des médiateurs externes pourrait donc mettre fin à la violence. Toutefois, cette solution se heurte à un obstacle de taille - obstacle que Girard ne pointe pas assez -, en ce que la différence entre le caractère intérieur et extérieur de la médiation n'est pas opérant à l'heure de contenir la violence mimétique ${ }^{8}$. Certes, le fait de prendre comme modèle un médiateur externe ne nous met pas en concurrence avec lui, mais cela nous plonge de façon inéluctable dans une concurrence effrénée avec les autres individus le prenant eux aussi pour médiateur externe. Si nous sommes socialistes et prenons François Mitterrand comme modèle dans un désir de présidence de la République, nous ne nous heurterons certes pas au désir de Mitterrand, mais en revanche, nous rencontrerons sur le chemin de la réalisation de notre désir, ceux de tous les socialistes prenant eux aussi Mitterrand comme modèle dans un désir de Présidence. Ce problème bien connu en politique, mais aussi dans le monde académique, est celui de la guerre des héritiers ou des fils spirituels, guerre dont nous pouvons apprécier l'intensité de la violence au quotidien. D'ailleurs, lorsque Girard fait de l'imitatio Christi, et de son imitation d'imitation, la seule sortie possible à la

8 Nous pourrions d'ailleurs, avec Derrida, mettre en doute la possibilité même de distinguer d'une façon générale entre un intérieur et un extérieur, comme nous le montrent les concepts de parasite, d'exergue, de titre, de marge ou encore de greffe (Derrida, 1980). 
violence mimétique' ${ }^{9}$ elle se heurte elle aussi au phénomène de la concurence des héritiers. Il suffit pour s'en convaincre de se rappeler combien l'Histoire du christianisme est grosse de luttes intestines (Armogathe, 2011), entre les groupes chrétiens, afin de savoir lequel d'entre eux imite au mieux le Christ et en est donc l'héritier le plus légitime ${ }^{10}$. Le travail sur le pôle « médiateur »du triangle mimétique est donc lui aussi un échec parce que le médiateur externe ne stoppe pas la violence mimétique mais ne fait que la déplacer, la différer, au niveau des individus se donnant un même modèle. Il diffère une impossible violence verticale en une violence horizontale généralisée.

Reste donc le pôle du sujet et de son désir. La solution dans ce cas serait de penser un sujet qui renonce à son désir, qui renonce à soi-même afin de ne jamais entrer dans la spirale mimétique. Cette solution se trouve aussi dans les textes de Girard : « Dès que le sujet perçoit le rôle de l'imitation dans son propre désir il doit renoncer au désir ou renoncer à son orgueil. La lucidité moderne déplace et élargit le problème de l'ascèse. Il ne s'agit plus de renoncer momentanément à l'objet pour mieux le posséder, mais de renoncer au désir lui-même. » (Girard, 1961, p. 257). Nous trouvons dans cette option une des sources du tournant levinassien de la pensée de Girard tel qu'il se dessine dans Achever Clausewitz. Toutefois, outre que comme le signale Girard lui-même, nous ne pouvons pas penser que cette conversion à l'oubli de $\operatorname{soi}^{11}$ frappera la totalité de l'humanité, l'oubli de soi peut être source d'encore plus de violence. Comme l'a montré JeanPierre Dupuy dans ses analyses du phénomène terroriste (Dupuy, 2002), l'oubli de soi que nous trouvons dans l'abnégation du Christ peut être perverti dans l'oubli de soi du kamikaze qui souhaite tuer en renonçant à se soucier de sa propre vie. Le seul oubli de soi ne permet donc pas de garantir la paix et peut, bien

9 «Ce que Jésus nous invite à imiter c'est son propre désir, c'est l'élan qui le dirige lui, Jésus, vers le but qu'il s'est fixé : ressembler le plus possible à Dieu le Père. » (Girard, 1999, p. 31).

10 Ceci permet d'expliquer, comme ne cesse de le rappeler Girard, que les guerres de religions sont bien plus violentes au sein d'une même religion qu'entre deux groupes religieux radicalement différents.

11 Nous parlons d'oubli de soi parce que Levinas inverse le précepte delphique et pose une éthique fondée sur le seul principe : « Ignore-toi - toi-même. » (Levinas, 2009, p. 279). 
au contraire, la renforcer. Même le renoncement au désir et l'oubli de soi peuvent être détournés au profit de la violence.

Le système de Girard semble donc fermé sur la violence apocalyptique parce que nous ne pouvons avoir aucun impact sur les pôles du triangle mimétique. Aucun des sommets du triangle n'ouvre la moindre brèche dans la forteresse de la violence totale. Toutefois, nous voyons là que la théorie mimétique est basée sur le fait que le désir commence toujours par un désir d’objet avant que de devenir un désir d'être, et c'est précisément cela que nous devons questionner maintenant afin de voir s'il n'y a pas une ouverture dans la clôture de la violence, non pas sur l'un des pôles du désir, mais sur le point de départ objectal du désir ainsi que dans son passage de l'objet à l'être.

\section{De l'objet à l'être : un désir onto-théo-logique}

UNE DES CRITILUES que nous pouvons adresser à Girard et à la théorie mimétique est d'en être resté, en un sens que nous allons préciser, à la grande tradition métaphysique $^{12}$. Cela est vrai du point de vue du champ sémantique, mais nous le retrouvons aussi dans toute la logique du système. De toutes parts, la théorie mimétique baigne dans la métaphysique. D’abord parce que Girard lui-même n'a de cesse d'insister sur la vérité proprement métaphysique du désir mimétique et sur la révélation exclusivement romanesque de cette vérité : « Le souci fondamental du romancier n'est pas la création de personnages, c'est la révélation du désir métaphysique. » (Girard, 1961, p. 169). Mais surtout, parce que lorsque ce désir métaphysique se fait jour, c'est parce que le désir du sujet ne se concentre plus tant sur l'objet afin de le posséder, que sur l'être du médiateur afin de le devenir : « L'objet n'est qu'un moyen d'atteindre le médiateur. C'est l'être de ce médiateur que vise le désir. » (Girard, 1961, p. 76). Le passage à la métaphysique, chez Girard, se fait de façon tout à fait classique par un saut de l'objet à l'être. Nous retrouvons bien dans ce glissement le fantasme métaphysique le plus

12 Certes, il ne s'agit pas ici de dire que Girard est un métaphysicien, mais que son système s'inscrit, peut-être malgré lui, dans la tradition de pensée métaphysique. Cela explique d'ailleurs sa tentation levinassienne dans Achever Clausewitz, voyant de façon tout à fait traditionnelle dans cet auteur, une sortie possible de la métaphysique (Levinas, 1974). 
fondamental et le plus classique dans l'Histoire de la philosophie ${ }^{13}$, fantasme selon lequel, ce que poursuivent les hommes doit avant tout se penser selon la catégorie de l'être. La vérité de tout désir est que tout désir mimétique est in fine un désir d'être. D’ailleurs, nous pouvons pousser encore plus loin sur le chemin de la métaphysique puisque, comme l'affirme Girard, derrière cet être (du médiateur) que nous désirons, se cache toujours déjà un désir d'Être. De l'être à l'Être la conclusion est métaphysiquement ainsi que mimétiquement bonne. Nous désirons lêtre du médiateur parce que nous pensons que celui-ci est autonome, pleinement maître de son propre désir. Nous croyons que le médiateur, lui, est une origine pure et vierge de son désir. Nous envions donc l'être du médiateur parce que nous nous le représentons comme causa sui. C'est donc bien son caractère divin que nous envions : « c'est le mensonge qu'entretien le désir triangulaire. Le héros se tourne passionnément vers cet Autre qui semble jouir, lui, de l'héritage divin. » (Girard, 1961, p. 80). Le médiateur, lui, ne semble influencé par personne, il semble n'imiter personne et être pleinement maître de lui. Cela apparait de façon particulièrement explicite lorsque Girard analyse l'indifférence dont fait preuve le médiateur à l'égard du sujet qui l'imite : «L'indifférent semble toujours posséder cette maîtrise radieuse dont nous cherchons tous le secret. Il paraît vivre en circuit fermé, jouissant de son être, dans une béatitude que rien ne peut troubler. Il est Dieu... » (Girard, 1961, p. 121). Sur ce chemin totalement métaphysique, la subjectivité romantique n'est, au niveau du désir, ni plus ni moins qu’une onto-théo-logie du sujet, celui-ci y étant pensé à la fois, comme étant, origine et cause de soi ${ }^{14}$. Aussi, le problème fondamental pour Girard est

13 Il suffit pour s'en persuader de voir comment Ferdinand Alquié explique la déception métaphysique de Descartes par le fait que celui-ci, s'en tenant aux objets, n'a pas trouvé l'être (Alquié, 1950).

14 Nous retrouvons en effet dans ce sujet romantique toutes les déterminations d'une pensée métaphysique et onto-théo-logique : « L'onto-théo-logie se définit donc selon des caractères extrêmement précis, sans lesquels on ne saurait identifier une pensée comme métaphysique : (i) « Le Dieu » doit s'inscrire explicitement dans le champ de la métaphysique, c'est-à-dire se laisser déterminer à partir d'une des déterminations historiques de l'être en tant qu'étant, éventuellement à partir du concept d'étant; (ii) il doit y assurer une fondation causale (Begründung) de tous les étants communs dont il rend raison ; (iii) il doit, pour ce faire, assumer toujours la fonction et éventuellement le nom de causa sui, c'est-à-dire de l'étant suprêmement fondateur parce que suprêmement fondé par lui-même. » (Marion, 1982, p. 287). 
que tout le système romantique qui porte le désir mimétique, repose sur une conception erronée, parce que métaphysique, de la divinité. C'est une transcendance déviée, ou, pour le dire dans les termes de la théologie, le sujet romantique confond la divinité et l'idole, dans une confusion de la verticalité et de l'horizontalité. D'où l'exergue de Mensonge romantique et vérité romanesque, qui pose, dans une citation de Scheler, la dichotomie fondamentale dans laquelle nous plonge le désir mimétique ainsi que l'essentiel de la thèse de Girard (Girard, 1961, p. 33). Ce serait donc l'idole qui ferme le système girardien, le médiateur pensé comme idole. Il nous faut donc voir avec Marion si nous ne pouvons pas dépasser le modèle de l'idole, et ce que cela implique tant du point de vue d'une possible sortie de la métaphysique que de la pensée du désir comme désir d'objet. En questionnant l'idole, nous pourrons remonter jusquà l'objet et ouvrir la théorie mimétique à une solution mettant fin à la violence dans l'humanité. Ainsi, en arrachant la théorie mimétique à sa conception idolatrique du désir, nous pourrons montrer quen la complétant par la phénoménologie de la donation, elle pourrait proposer un moyen efficace de lutter contre la violence.

La confusion de la divinité et de l'idole, ou de la pensée idolâtrique de la divinité, est au cœur de la pensée de Jean-Luc Marion, et elle nous intéresse ici au plus haut point parce qu'elle a des résonances en termes d'objet et de dépassement vers l'être. Toute la phénoménologie de la donation de Marion vise à montrer que jusque lors, tant la modernité cartésienne ${ }^{15}$ que la révolution critique $^{16}$ ont privilégié le modèle de l'objet (et donc de la construction) dans la phénoménalité. Ne pouvait apparaître et donc faire l'objet d'une expérience, que ce qui se donnait sur le mode de l'objectité, c'est-à-dire ce qui pouvait entrer dans des conditions de possibilité dictées par un sujet ${ }^{17}$. Même Husserl et Heidegger, qui se sont pourtant arrachés à l'objectité par la pensée d'un phénomène

15 C'est là toute la thèse de l'ontologie grise (Marion, 1975).

16 « Il y a deux conditions sous lesquelles seulement la connaissance d'un objet est possible, premièrement l'intuition, par laquelle il est donné, mais seulement comme phénomène : deuxièmement le concept, par lequel est pensé un objet, qui correspond à cette intuition. » (Kant, 1980, p. 848).

17 « Ce qui s'accorde avec les conditions formelles de l'expérience (quant à l'intuition et aux concepts) est possible. » (Kant, 1980, p. 948). 
se donnant en lui-même et à partir de lui-même ${ }^{18}$, n’ont pas réussi à échapper à cette idée selon laquelle ce qui nous apparaît est déterminé par les conditions de possibilité d'apparaître que lui dicte un sujet percevant ${ }^{19}$. La phénoménologie allemande a certes dépassé le modèle moderne de la construction des objets par celui de la constitution des phénomènes, mais il n'en reste pas moins que l'apparaître des phénomènes y demeure déterminé par les conditions de possibilités dictées par un sujet. D'où la question de Marion : « l'expérience se borne-t-elle à faire expérimenter des objets, ou, au contraire, la constitution d'objets n'en définitelle pas seulement un champ particulier et restreint, qui contredit l'immense banalité de la saturation intuitive de la phénoménalité ? 》(Marion, 2005a, p. 168). D’ailleurs, ce modèle privilégié de la phénoménalité (conditionné par le sujet percevant) a été mis à mal dès sa formation, c'est-à-dire, au moins depuis Descartes. Tant Descartes que Kant, Husserl ou même Heidegger ont pensé, aux marges de cette phénoménalité indexée sur un sujet - au-delà de cette phénoménalité objectale donc -, un autre type de phénomènes qui, loin d'être constitués par un sujet, s'imposent à lui. C'est le cas de l'idée d'infini chez Descartes qui précède, dans la troisième méditation, toute construction de notre part ${ }^{20}$, ou encore des idées esthétiques chez Kant qui débordent tout concept que peut en former le sujet ${ }^{21}$. Pour décrire chacun de ces phénomènes, nous avons besoin de dépasser la logique de l'objet parce qu' ils s'imposent au sujet malgré lui, avant même qu'il n'ait pu les cercler dans un concept leur permettant d'apparaître. "Il y a des phénomènes, dits 'saturés', pour lesquelles l'intuition qui se donne excède le concept non seulement que nous avons mais même que nous pouvons en avoir.

18 «Comme signification de l'expression 'phénomène', il faut donc s'en tenir à : le se-montrant-de-soimême, le manifeste, [...] le se-montrant-par-soi-même. » (Heidegger, 1986, pp. 55-58).

19 «Husserl recule en deçà de sa propre avancée, en restreignant la donation à l'une de ses moindres possibilités phénoménologiques, l'objet. » (Marion, 1997, p. 50). Quant à Heidegger : « il recule lui aussi devant l'originarité de la donation. » (Marion, 1997, p. 51).

$20 \ll[\ldots]$, je n'aurais pas néanmoins l'idée d'une substance infinie, moi qui suis un être fini, si elle n'avait été mise en moi par quelque substance qui fût véritablement infinie. » (Descartes, 1992, p. 445).

21 « Une Idée esthétique ne peut devenir une connaissance, parce qu'elle est une intuition (de l'imagination), pour qui on ne peut jamais trouver un concept adéquat. » (Kant, 1985, p. 1131). 
Marion ne prétend certes pas être le premier à décrire ces phénomènes ${ }^{22}$, mais il est le premier à en faire le centre de toute phénoménalité. Il y aurait d'un côté des objets, ou des phénomènes de droit commun, construits ou constitués par un sujet, à sa mesure et à son image donc; de l'autre, des phénomènes saturés qui s'imposent à lui et lui en imposent (Vinolo, 2016).

Cette dichotomie résonne et raisonne dans la problématique de la divinité. Nous la posons d'abord chez Marion avant que de l'étendre à la divinité idolâtrique telle que nous l'avons vue se mettre en place dans la théorie du désir mimétique. Cette logique de la dichotomie des « phénomènes de droit commun » et des « phénomènes saturés » frappe de plein fouet Dieu lui-même. Elle se retrouve dans les deux phénomènes que sont l'idole et l'icône. Celles-ci ne relèvent pas de deux types d'objets différents puisqu'un même objet peut être successivement idole et icône, mais bien de deux façons d'apparaître, selon la réception qu’en fait le sujet : « Icône et idole indiquent une manière d'être des étants, ou du moins de certains d'entre eux. » (Marion, 1982, p. 15). Or, ces deux manières de recevoir le divin ont quelque chose en commun et pourtant doivent être tenues à distance. Elles ont en commun qu'elles se donnent toutes deux comme faisant signe vers autre chose qu'elles-mêmes. Comme nous le montre cette question au niveau purement esthétique, tant les icônes que les idoles sont des œuvres qui népuisent pas toutes seules la totalité de ce qu'elles donnent à voir, elles sont toujours déjà une ouverture sur autre chose qu'elles-mêmes : « ne peuvent prétendre aux statuts contradictoires d'idoles ou/et d'icônes que ces œuvres que l'art a ainsi œuvrées qu'elles ne bornent pas leur visibilité à elles-mêmes [...], mais, [...] font indissolublement signe vers un autre terme, [...]. » (Marion, 1982, pp. 1617). En ce sens, elles se distinguent radicalement des objets dont nous pouvons facilement épuiser la visibilité. En effet, quiconque a vu un enfant manipuler un objet technique constate qu'en quelques minutes il en a fait le tour, au point de pouvoir l'utiliser sans même devoir encore le regarder (Serres, 2012). En quelques minutes à peine, l'enfant a vu tout ce que cet objet donnait à voir. Il en va tout autrement des œuvres d'art, puisque comme nous l'expérimentons tous, il nous faut toujours retourner dans les musées afin de voir et revoir les tableaux,

22 « [...] c'est à Kant - penseur de la pénurie en intuition du phénomène commun - qu'il revient d'avoir pressenti ce que nous nommons un phénomène saturé. » (Marion, 1997, p. 277). 
comme si nous n'en avions jamais fini avec eux. Nous n'avons jamais fini de voir une œuvre d'art, comme si la source de visibilité dans laquelle elle prend racine était inépuisable (Vinolo, 2017). Ainsi, idole et icône nous donnent un autre mode de visibilité que celui de l'objet, un mode qui nous porte immédiatement vers celui de la visibilité qui fait signe vers autre chose quelle-même, et donc vers une visibilité ouverte, d'où leur place privilégiée dans les représentations de Dieu.

Mais, bien que nous puissions mettre en relation l'idole et l'icône en ce qu'elles font toutes deux « signe vers », l'idole se distingue de l'icône parce que cette façon de « faire signe vers » est radicalement différente dans les deux cas : « Il faudrait donc interroger les signa sur leur mode de faire signe, soupçonnant que l'idole et l'icône ne se distinguent qu'autant qu'elles font signe de manière différente. » (Marion, 1982, p. 17). L'immense différence est que si l'icône fait signe vers une altérité radicale, l'idole est un signe autoréférentiel, elle donne notre image parce quelle donne avant tout notre limite ${ }^{23}$. D'où le fait que l'idole soit aussi un problème éthique et pas uniquement esthétique, puisque ce que nous sommes capables de recevoir comme visibilité marque nos limites, non seulement pour ce qu'il en est de la perception externe mais aussi pour la perception interne. Cette clôture autoréférentielle de l'idole est particulièrement frappante dans le cas de l'idole pensée comme concept. En effet, s'il y a bien une idole de la divinité, c'est le concept métaphysique de Dieu. Nous pouvons même, avec Marion, et précédemment avec Pascal ${ }^{24}$, opposer le Dieu des philosophes à celui des religions. Toute la pensée de Dieu sans l'être de Marion pourrait d'ailleurs se cristalliser dans ce refus d'un concept de Dieu obsédé par l'être, afin de lui opposer la primauté d'un Dieu

23 « Mon idole définit ce que je peux supporter de phénoménalité - le maximum d'intensité intuitive que je puis endurer tout en gardant mon regard sur un spectacle distinctement visible, tout en transformant une intuition en visible distinct et constitué, sans défaillir dans la confusion ou l'aveuglement. Mon idole met ainsi à nu l'empan de toutes mes visées - ce que j'ambitionne de voir, donc aussi de vouloir voir et faire, bref elle dénude mon désir et mon espérance. Ce que je regarde de visible décide de qui je suis. Je suis ce que je peux regarder. Ce que j’admire me juge. » (Marion, 2001, p. 76).

24 Nous retrouvons cela dans le Mémorial (Pensées retranchées $n^{\circ} 711$ ) de Pascal, rédigé suite à son expérience mystique du 23 novembre 1654 : « Feu / Dieu d'Abraham, D’Isaac, Dieu de Jacob, / non des philosophes et des savants. / Certitude, certitude, sentiment de joie, paix. / Dieu de JésusChrist. » (Pascal, 2000, p. 851). 
de la charité : « Quand une pensée philosophique énonce, de ce qu’elle nomme alors « Dieu », un concept, ce concept fonctionne exactement comme une idole : [...]. » (Marion, 1982, p. 26). Or, nous comprenons facilement pourquoi le concept de Dieu nous amène immédiatement à la pensée de la divinité en tant qu'idole. Un concept, par définition, puisqu'il émane de la cognition humaine, ne peut pas aller au-delà de celle-ci : « Car le concept, quand il sait le divin dans son emprise, et donc nomme « Dieu », le définit. Le définit, donc aussi le mesure à la dimension de son emprise. » (Marion, 1982, p. 44). Le concept est comme l'idole, à la fois l'image et le miroir de ce que nous sommes. Sans aller jusquà l'exemple extrême de Spinoza selon lequel si les triangles avaient un Dieu, celui-ci aurait trois cotés ${ }^{25}$, nous comprenons que par définition, un concept, tout comme un objet, ne peut être quà mesure humaine, et reflète donc bien plus notre expérience du divin que la divinité elle-même : « Le concept consigne dans un signe ce que d'abord l'esprit avec lui saisit (concipere, capere); mais pareille saisie ne se mesure pas tant à l'ampleur du divin, quà la portée d'une capacitas, qui ne fixe le divin en un concept, tel ou tel, quau moment où une conception du divin la comble, donc l'apaise, l'arrête, la fige. » (Marion, 1982, p. 26). Lors, nous retrouvons logiquement chez Marion, ce que nous avions vu de l'idole comme miroir chez Girard. L'idole est une projection divine de l'Homme, de façon symétrique, comme dans un miroir. Pour ce, l'idole est un Dieu spectral, parce qu'une image de l'Homme s'attarde en lui : « L'idole atteste en effet le divin, au point de vue de la visée qui le produit comme son reflet. » (Marion, 1982, p. 43). Elle fait donc certes signe, mais ce vers quoi elle fait signe nous plonge dans une boucle autoréférentielle et close. Nous retrouvons là, dans cette boucle autoréférentielle de l'idole, la boucle mimétique qui est, elle aussi, autoréférentielle, puisqu'au moment de la médiation double, le médiateur est médiateur pour le sujet, mais le sujet est lui-même médiateur pour le médiateur. Nous retrouvons donc la même dénonciation de l'idole chez Girard et Marion selon la même logique de la projection de soi dans l'idole, projection qui coupe court à toute expérience de l'altérité

$25 \ll[. .$.$] je crois que le triangle, s'il était doué de langage, dirait en même manière que Dieu est$ triangulaire éminemment et le cercle que la nature de Dieu est circulaire éminemment. De même n'importe quel être affirmerait de Dieu ses propres attributs, se rendrait semblable à Dieu et toute autre manière d'être lui paraîtrait laide. » (Spinoza, 1966, p. 298). 
radicale ou de toute extériorité véritable que suppose le divin. La boucle autoréférentielle de l'idole est responsable de la clôture radicale parce que, comme nous le savons depuis Girard, la logique de la réciprocité (Anspach, 2002) - et donc de l'image qui rend, dans un miroir, ce qu'on commence par lui donner - est celle de la violence. Contre l'idole qui rend (ne serait-ce qu'un reflet) il faudrait donc penser une image qui donne. Peut-être est-ce là le secret de l'icône ?

Contrairement à ce qu'il en est pour l'idole, nous ne pouvons com-prendre l'icône dans un concept puisque ce vers quoi elle fait signe ne se laisse pas saisir par un concept et le déborde toujours-déjà. Le concept obéissant aux limites de ce qu'un esprit humain peut rassembler, il ne peut y avoir de mise en concept de ce qui nous dépasse et nous excède. C'est le cas de ce vers quoi fait signe l'icône, puisquà la différence de l'idole, elle ne fait pas signe vers le sujet dans une boucle autoréférentielle, mais vers une altérité radicalement autre et donc inaccessible, vers une altérité non-objectivable parce que précisément non-objectale. Nous pourrions certes imaginer un concept qui puisse convenir à l'icône, mais il faudrait pour ce faire, qu'il renonce à saisir l'insaisissable : « [...] l'icône peut procéder aussi conceptuellement, pourvu du moins que le concept renonce à comprendre l'incompréhensible, pour tenter de le concevoir, donc aussi de le recevoir à sa propre démesure. » (Marion, 1982, p. 35). Or, ce concept existe, mais c'est un concept qui se place dans la non-saisie, et donc dans la distance : « Nous retrouvons, à l'œuvre dans l'icône, le concept de distance : que l'union croît à la mesure de la distinction, et réciproquement. » (Marion, 1982, p. 36). Ce concept est de la plus grande importance parce qu' il est aussi en jeu dans toute la théorie de la violence mimétique, ne serait-ce que dans la variation de la distance que suppose la position interne ou externe du médiateur, position qui détermine en grande partie, pour Girard, le surgissement de la violence. Nous retrouvons aussi cette importance de la distance dans le fait que, pour Girard, l'absence de distance provoque la violence plus encore que la distance elle-même. Mais à la différence du médiateur externe qui peut changer de position et nous mettre en rivalité avec d'autres sujets le prenant comme médiateur, la distance dont il est question dans l'icône est infranchissable. Par définition, il n'y a pas de changement de position possible de l'icône, sans quoi elle deviendrait une idole. Ce vers quoi fait signe l'icône est par définition inatteignable. 
Nous avons ainsi chez Marion, comme chez Girard, une clôture portée principalement par le passage de l'idole à l'icône. Cette clôture se donne parce que l'idole est une boucle autoréférentielle construite par le sujet et son reflet. Que nous l'appelions « médiation double » chez Girard ou anthropomorphisme divin chez Marion ${ }^{26}$, dans les deux cas, la fermeture provient du fait que là où nous devrions penser une distance, nous avons en réalité une absence de distance, et plus précisément un miroir. La structure de l'objet est principalement en cause dans la violence puisque l'objet est ce qui, étant construit à notre mesure, ne peut nous mener que vers l'idole, c'est-à-dire vers nous-même : « toute image doit reproduire en image la mesure d'un désir ; c'est-à-dire que toute image doit se faire l'idole de son voyeur. » (Marion, 1991, p. 92). Nous devons donc envisager la possibilité d'un dépassement de l'objet non pas vers l'idole et son être, mais vers l'icône. C'est à cette seule condition que nous pourrons trouver une brèche dans la violence mimétique, ouvrant la boucle autoréférentielle sur un point de fuite non-idolâtrique nous permettant de nous défaire de la structure du miroir.

\section{Désirer au-delà de l'être : le signe contre l'image}

LA CLÔTURE MÉTAPHYSIQUE est indépassable dans la logique girardienne parce que même la solution levinassienne de l'oubli de soi ou du renoncement au désir peut être pervertie au profit de la violence. Mais qu’en serait-il si le dépassement de l'objet ne se faisait pas en direction de lêtre, mais en direction de quelque chose qui échappe à l'être, de quelque chose qui se soustrait à la question de l'être ? Que deviendrait la clôture métaphysique de la théorie mimétique si le désir ne se fixait pas premièrement sur des objets, ce qui bloquerait à la source son cheminement vers un désir d'être ? C'est encore la phénoménologie de Marion et plus particulièrement sa catégorie de « phénomène saturé » qui nous permet de mettre la théorie mimétique au risque d'un désir de l'autre-que-l'objet et donc aussi de l'autre-que-l'être, par la ligne de fuite qu'elle trace hors de l'objectité.

Si la clôture se fait par l'objet pensé comme idole, nous ne pouvons faire l'économie de son lien avec l'image. En effet, l'idole fait signe vers une présence

26 «Dans l'idole, se trahit et s'étalonne ainsi la fonction divine du Dasein. » (Marion, 1982, p. 42). 
de soi dans une boucle autoréférentielle, parce que sa construction, dont nous avons vu quelle obéit à la logique du miroir, se fait selon le paradigme de l'image. Il y a ainsi un lien inextricable entre l'objet, l'image et l'idole. Or, ces trois concepts nous mènent tout naturellement vers la présence et donc l'être. Se mettre à distance de l'être et de la présence implique donc de questionner le modèle de l'image, pour tout au moins distinguer différents types d'images, comme nous pouvons distinguer l'idole de l'icône. L'image, par définition, présente ou re-présente quelque chose. Mais que ce soit de façon directe ou par un redoublement, l'image est liée de façon essentielle au présent, et donc à l'être. Comme le remarque fort justement Charles Ramond (2002) ${ }^{27}$, il y a un certain type d'objets auquel l'image n'a pas accès. Elle ne peut ni les présenter ni même les re-présenter. Ces objets sont les négations et les absences. Nulle image, aussi originale, torturée ou magnifique soit-elle, ne peut présenter une négation ni une absence. Imaginons aujourd'hui un dessin, une peinture, ou même une photo de Manhattan. Nul ne peut voir sur cette image que les deux tours jumelles du World Trade Center sont absentes, parce que de fait, visuellement, elles ne manquent pas. Elles ne manquent pas dans ce que l'image donne à voir. L'image est pleine, totalement présente, donnant tout ce qu'elle peut donner à voir. Seul un discours (comparant par exemple deux images de New York avant et après 2001) peut exprimer l'absence ou la négation. L'image, quant à elle, est prisonnière de la présence et donc de l'être.

Or, c'est selon la logique de l'image que se transforme l'objet du désir mimétique, et que celui-ci passe logiquement de l'objet à lêtre. À de nombreuses reprises, Girard parle en termes d'images. Son modèle est envahi par le modèle visuel de l'image et du regard. Nous le voyons dans ses analyses du romancier : « Le romancier n'est pas un réaliste de l'objet mais il est un réaliste du désir. Les images doivent "transfigurer" l'objet. » (Girard, 1961, p. 97). Mais nous retrouvons cela à un niveau plus fondamental de l'apparaître de l'objet qui est lui-même totalement déterminé par la somme des regards qui se posent sur lui : « La valeur de l'objet consommé ne dépend plus que du regard de l'Autre. Seul le désir de

27 Nous noterons d'ailleurs que par cette impossibilité de l'image à présenter des absences ou des négations, Charles Ramond questionne cette sensation quasi immédiate que nous avons, que les rêves sont faits d'images, puisque de fait, les rêves sont pleins de négations et d'absences. 
l'Autre peut engendrer le désir. » (Girard, 1961, p. 217). L'objet apparaît désirable à mesure que d'autres regards se posent sur lui. Nous désirons, chez Girard, avant tout ce que nous voyons, et l'image de l'objet est totalement déterminée par des regards, et donc encore par des modèles visuels. D’ailleurs, lorsque Girard parle de la modification de l'objet par les regards qui se posent sur lui, c'est encore en termes de trans-figuration qu'il s'exprime, alors que nous aurions pu imaginer, dans des paradigmes non-visuels, que Girard s'exprime avec le vocabulaire de la transsubstantiation ou de la modification de sa signification. Dans le vocabulaire de Girard, c'est bien la figure de l'objet qui se modifie - et donc ni sa substance ni son sens : « Le désir triangulaire est le désir qui transfigure son objet. » (Girard, 1961, p. 47). Enfin, lorsque Girard résume la théorie mimétique telle qu'elle se donne du point de vue du héros, c'est encore l'imagination - et donc une certaine conception de l'image - qui pointe : « C'est la transfiguration de l'objet désiré qui définit l'unité de la médiation externe et de la médiation interne. L'imagination du héros est la mère de l'illusion mais il faut encore un père à cet enfant et ce père est le médiateur. » (Girard, 1961, p. 51). De toutes parts donc, la théorie mimétique est inondée par le modèle visuel, et nous comprenons alors pourquoi et dans quelle mesure elle demeure prisonnière de la métaphysique de la présence.

Toutefois, diront les lecteurs de Girard, ce modèle objectal, visuel et idolâtrique est justement celui que Girard dénonce, et certainement pas celui qu'il propose. Ce modèle est celui que la théorie mimétique met au jour dans le seul but de le mettre à mal, étant donné que sa simple monstration le déconstruit. Au contraire, afin de sortir de ce modèle, Girard propose un tout autre modèle du désir, modèle non-idolâtrique, qui ne se focalise pas sur la transcendance déviée mais sur la transcendance christique et donc réelle. C'est là la solution de l'imitatio Christi, solution qui devrait nous permettre de sortir de la violence mimétique en rompant la boucle autoréférentielle de l'idole par l'ouverture d'un modèle s'assumant lui-même comme imitateur. En effet, pour Girard, l'imitation du Christ est la solution à la violence mimétique parce que le Christ n'est pas un modèle comme les autres en ce qu'il assume ne pas être la source de ses désirs. Il ne se pose pas comme causa sui de ceux-ci - comme le font au contraire les médiateurs humains -, mais affirme imiter le désir du Père : «L'invitation à imiter le désir de Jésus peut sembler paradoxale car Jésus ne prétend pas posséder de 
désir propre, de désir "bien à lui". » (Girard, 1999, p. 31). Nous comprenons alors dans quelle mesure cette imitatio Christi permet de rompre la boucle autoréférentielle en ce que loin de nous renvoyer notre image dans un reflet, le désir du Christ, présenté par lui-même comme mimétique, nous ouvre immédiatement sur le Père en mettant à mal le Christ comme source de son désir, et donc aussi comme source de l'originalité de son être. Nous retrouvons là la rupture des deux caractéristiques fondamentales des médiateurs idolâtriques parce que le Christ n'est ni l'origine de ses désirs ni celle de son être : « Contrairement à ce que nous prétendons nous-mêmes, il ne prétend pas "être lui-même", il ne se flatte pas de "n’obéir qu’à son propre désir". » (Girard, 1999, p. 31). Néanmoins, dans son imitation du Père, le Christ lui-même demeure prisonnier du modèle visuel, de l'image et donc de la métaphysique de la présence et de l'être. Lorsque Girard pense la relation entre le Christ et le Père, c'est encore et toujours le modèle de l'image qui se fait jour : « Son but [au Christ] est de devenir l'image parfaite de Dieu. » (Girard, 1961, p. 31). Cette citation est d'autant plus surprenante que c'est Girard lui-même qui souligne le mot « image », montrant par-là que ce mot a été pleinement pensé et pesé.

Or, cette relation Christ-Père (ou fils-Père de façon générale, puisque cette relation peut être étendue à toute la lecture de l'OEdipe que donne Girard) est doublement problématique en ce que tout d'abord, le Christ ne cherche pas à devenir l'image du Père. Au contraire, loin de montrer le Père, le Christ le signifie. Qui rentre dans une église et voit le Christ en croix ne voit pas Dieu le Père en ce que le crucifix ne présente ni ne re-présente Dieu le Père, il le signifie. Mais surtout, en en restant encore une fois au modèle de l'image, la théorie mimétique, même dans le paradigme de l'imitatio Christi, ne peut pas totalement ouvrir la boucle autoréférentielle de l'idole parce que même la pensée de la vraie transcendance demeure prisonnière de l'image, et donc de la présence et de l'être, parce que si l'absence peut être signifiée, ce qui se montre se limite au domaine de la présence et donc de l'être. Nous retrouvons là la lutte millénaire - dont Derrida (1967) a montré les conséquences - entre le modèle de la vue et celui de l'écrit, ou de la monstration contre la signification. Ainsi, touchonsnous le problème fondamental de la théorie mimétique. Elle ne peut sortir de la violence mimétique parce qu'elle ne peut sortir de l'idole. Non pas parce que Girard s'en tient à la transcendance déviée idolâtrique, mais parce que, tout étant 
pensé selon le paradigme de l'image, le passage de l'objet à l'être se fait naturellement par le lien inextricable qui unit toute image à la présence. Mais nous pouvons alors penser un dépassement de la théorie mimétique, ou sa relève, dans une pensée qui s'arracherait au paradigme visuel afin de lui substituer un modèle linguistique. Ou bien nous sommes capables de penser une image qui signifie plus qu'elle ne montre, ou bien il nous faut tout simplement abandonner le paradigme de l'image afin de mettre à mal son corrélat inévitable en ce qui concerne la métaphysique de la présence, et donc la violence.

Loin de rejeter toute image, nous pouvons changer de paradigme dans la pensée de l'image et en passer paradoxalement à une image qui ne montre presque rien, mais qui signifie. Il y a une image qui fonctionne sur le modèle du signe linguistique plus que sur celui de la représentation. Cette image qui signifie plus qu'elle ne montre est l'icône telle que la pense Marion. L'idole, en effet, de par le mimétisme selon la logique de la copie et de l'image dans lequel elle se perd, instaure une concurrence inévitable entre le modèle et la copie. La copie cherche toujours à s'accaparer le privilège de l'original et, dans un mouvement de supplémentarité, à le remplacer. Le propre de tout supplément ou de tout représentant (lieu-tenant) est de tellement « tenir lieu de » qu'il finit par «se substituer à », rendant la concurrence et la rivalité inévitables : « Le supplément s’ajoute, il est un surplus, une plénitude enrichissant une autre plénitude, le comble de la présence. [...] Mais le supplément supplée. Il ne s'ajoute que pour remplacer. Il intervient ou s'insinue à-la-place-de ; [...]. » (Derrida, 1967, p. 208). Marion est parfaitement conscient de ce problème et propose l'icône précisément afin de sortir de ce paradigme de l'image en tant qu'elle entre en concurrence avec ce dont elle est l'image. La croisée du visible est parfaitement clair sur ce point. Nous pouvons y lire que dans le domaine de l'idole : « l'imitation tente toujours de ravir à un modèle aussi peu que ce soit de sa perfection, pour s'en glorifier autant que possible ; [...]. » (Marion, 1991, p. 136). Ainsi, la rivalité est essentielle à l'image pensée selon la logique de l'idole parce que plus l'image se parfait, plus elle entre en concurrence avec ce dont elle est l'image. D’où la dénonciation pascalienne de sa vanité dans la Pensée 37 : « Quelle vanité que la peinture qui attire l'admiration pour la ressemblance des choses dont on n'admire point les originaux! »(Pascal, 2000, p. 551). En revanche, l'icône n'entre pas en concurrence avec ce qu'elle représente parce que justement elle ne représente rien, mais 
signifie. Elle renonce à remplacer ce qu'elle signifie par ce qui est, et il est tout à fait notable dans le cadre de notre discussion des thèses de Girard, qu'elle porte un renoncement explicite à la « rivalité mimétique » : « Elle [l'icône] renonce, par ascèse esthétique, à la rivalité mimétique de la gloire ; [...] l'icône [...] ne se glorifie que de remettre toute gloire à l'invisible. » (Marion, 1991, p. 136). Parce qu'il ne représente pas le Père, pas plus que le crucifix ne représente le Christ, le jeu de miroir qui sétait mis en place, dans l'idole, entre le modèle et la copie, peut être parasité : « L'icône commune n'imite donc pas plus la Croix que la Croix n'imite la sainteté invisible ; l'icône commune répète la transition du visible à l'invisible qui fait de la Croix le signe de gloire du Saint. » (Marion, 1991, p. 133). Ici, Marion nous permet de compléter Girard. Alors que Girard pense la relation mimétique entre le Christ et le Père selon le modèle de l'image, Marion refuse systématiquement de penser cette relation selon la catégorie de la représentation pour lui préférer le modèle de la signification : « l'icône s'accomplit en renonçant définitivement à prétendre imiter le Saint auquel elle se remet et s'en remet ; si une convenance lui revenait, ce serait par grâce imméritée et en vertu d'une communion de volonté, jamais par la suffisance d'une correspondance intrinsèque; [...]. » (Marion, 1991, p. 136).

Nous avons donc, au cœur même de la relation entre le modèle et l'imitateur, une première relation close et idolâtrique qui se fait, chez Girard, sur le modèle de l'image. Mais Marion nous permet de penser cette relation, grâce à l'icône, non plus en termes de ressemblance mais de reconnaissance, c'est-à-dire, in fine, de signification : « L'icône répète, au degré le plus disséminé, le renvoi de la sainteté au Saint ; [...]. » (Marion, 1991, p. 135). La théorie mimétique ne prend donc pas suffisamment en compte le fait qu'il y a deux types d'itérations, celle de l'idole qu'elle utilise abondamment, et celle de l'icône qui échappe à la logique mimétique parce quelle échappe à la logique du redoublement et de la suppléance de toute image : «Une telle répétition [celle de l'icône] rompt ici avec toute imitation, puisque celle-ci [celle de l'idole] va du visible au visible par ressemblance, tandis que celle-là va du visible à l'invisible par reconnaissance. » (Marion, 1991, p. 132). Nous pouvons donc essayer de penser la relation entre le sujet et le médiateur non plus en termes de ressemblance mais de reconnaissance.

Dans cette opposition, c'est encore la visibilité qui est en jeu, et plus précisément l'invisibilité. Paradoxalement, l'icône se met en place par le regard qu'elle 
porte. Mais ce regard n'est pas celui que le spectateur porte sur elle; c'est le regard qui, depuis la toile ou le bois, nous envisage. Or, ce que nous voyons dans ce regard que porte sur nous l'icône, ce sont principalement les pupilles, c'est-à-dire deux points noirs. L'icône est donc faite par un regard qui littéralement ne se porte sur aucun visible. En un sens, l'icône est transparente : « l'icône se proclame elle-même serviteur inutile d'une vénération qu'elle ne touche pas, mais devant laquelle elle s'efface jusquà la transparence. » (Marion, 1991, p. 139). Mais en plus d'attirer notre regard sur deux points noirs sur lesquels il n'y a rien à voir, ce vers quoi elle fait signe est aussi invisible. L'icône, contrairement à l'idole, ne cherche pas à porter dans le visible l'invisible divin, mais à le conserver invisible tout en le signifiant. Elle cherche donc à faire signe vers un invisible afin de le porter sans le montrer : « Nous devons donc comprendre comment le Christ Jésus offre non une image seulement visible du Père restant invisible, mais bien un visage (visible) de l'invisible lui-même (le Père), une image visible de l'invisible en tant qu'invisible. » (Marion, 1991 : 104). Plutôt donc que de désirer des objets visibles dont l'apparaître est déterminé par la concentration des désirs, nous pouvons désirer des objets qui ne sont pas construits par la logique de la monstration mais par celle de la signification. Dans la phénoménologie de Marion, cette logique est portée par les phénomènes saturés. Plutôt que de désirer des objets qui nous amènent de façon inévitable vers lềtre, ou que de nous focaliser sur un médiateur lui-même image (et donc ressemblance rivale) de la transcendance, empruntons avec Marion le chemin du désir des phénomènes saturés qui nous portera vers l'indifférence au discours sur l'être et donc vers une sortie possible de la violence de l'idolâtrie.

Là où les objets se construisent par remplissement partiel ou total du concept par ce qui se donne dans l'intuition, les phénomènes saturés apparaissent par excès d'intuition sur le concept. Toutefois, dans l'immense monde d'objets qui nous entoure depuis la modernité et son obsession quantitative (Rey, 2016), nous pourrions nous faire l'objection selon laquelle si avec Marion, nous nous condamnons à ne désirer que des phénomènes saturés et non plus des objets, nous sommes limités à l'extrême dans l'éventail de tout ce que nous pourrions désirer. Cette objection qui nous pousserait vers une ascèse extrême nous permettant de ne désirer que ce qui est extrêmement rare, ne tient toutefois qu'à la seule condition que les phénomènes saturés soient rares. Ainsi, si ce que nous 
propose Marion afin de sortir de la violence mimétique est de ne faire porter notre désir que sur les icônes, pas plus que chez Girard, la majorité des Hommes ne se soumettra à ce désir pauvre.

Néanmoins, comme l'écrit Marion dans un texte unique, les phénomènes saturés ne sont pas rares, mais au contraire banals (Marion, 2005a) ${ }^{28}$. Ils le sont avant tout parce qu'ils n'obéissent pas à la logique de la typologie qualitative mais, au contraire, à celle de l'herméneutique quantitative. Dans un premier moment de son œuvre, Marion a présenté la distinction entre les objets et les phénomènes saturés selon le vocabulaire de la typologique catégorielle, limitant ce faisant leur nombre. En plus de la multitude des objets qui nous entourent, il $\mathrm{y}$ a, autour de nous, quelques phénomènes saturés. Ce vocabulaire en termes de types est explicite dans Étant donné: «Il parait désormais possible de tracer, ne fût-ce qu'en esquisse encore, une topique des différents types de phénomènes. » (Marion, 1997, p. 309). Mais un deuxième moment de la phénoménologie de la donation séloigne de cette catégorisation discontinue des phénomènes afin de se livrer à une simple herméneutique continuiste entre tous les phénomènes, depuis les phénomènes pauvres en intuition jusqu'aux phénomènes saturés, en passant par les phénomènes de droit commun ou objets. Lors, dans cette logique continuiste, presque tous les phénomènes peuvent être reçus comme des phénomènes saturés parce que la saturation ne relève plus d'un type mais d'une modalité possible de leur réception par un sujet : « Devant la plupart des phénomènes, même les plus sommaires (la plupart des objets, produits de la technique et reproduits industriellement), s'ouvre la possibilité d'une double interprétation, qui ne dépend que des exigences de ma relation, toujours changeante à eux. » (Marion, 2005a, p. 156). Or, cette banalité ouvre une sortie possible à la violence mimétique puisque la solution ne serait plus de désirer certains non-objets, mais au contraire de pouvoir tout désirer, tant que nous portons sur ce que nous désirons, un regard non-objectal. Selon cette logique herméneutique, afin de faire obstacle à la violence mimétique, il faudrait non pas porter notre désir sur certains phénomènes non-objectaux - puisque cette non-objectalité ne relève pas

28 Nous avons commenté ce texte et les problèmes herméneutiques et typologiques qu'il pose à la phénoménologie de la donation dans Vinolo, 2016. 
d'un type de phénomènes -, mais au contraire porter un regard non-objectal sur tout ce que nous désirons.

Nous pouvons donner des exemples précis de cette double lecture possible afin de préciser la sortie de la violence mimétique. Nous ne prenons que l'exemple de l'ouie, de l'odorat, et du goût, mais ces analyses pourraient être étendues à la totalité de nos sens, c'est-à-dire à la totalité de ce qui nous apparait. Commençons par l'odorat. Nous pouvons imaginer deux façons de sentir un vin. Tout d'abord une façon objectale, qui vise à identifier ce que nous sentons, le classer, lui donner une identité claire et précise, c'est-à-dire à le construire comme un objet. C'est le cas lors des compétitions d'œnologie, lors desquelles les odeurs de vins ne sont perçues que dans le but d’être classées selon le cépage, le château et l'année. Là, le nez ne jouit point, il est totalement happé par le but de mettre un concept et une détermination sur ce qui se donne à lui. D’un autre côté, nous connaissons tous ces moments lors desquels nous nous abandonnons à une odeur, à un parfum, sans chercher à l'identifier, tout simplement pour en jouir. D'un côté nous avons une utilisation objectale du sens de l'odorat; de l'autre une perception saturée, qui ne suppose aucun concept préalable dans lequel prendre place. D'ailleurs, bien souvent, moins nous sommes capables de mettre des concepts sur ce que nous humons, plus nous en jouissons facilement. Au contraire, les spécialistes des métiers du nez, par déformation professionnelle, obsédés qu'ils sont par l'identification de ce qu'ils sentent, ne jouissent que très rarement de ce qu'ils sentent, parce qu'ils ne peuvent s'y abandonner totalement. D'un côté donc, il s'agit de plaquer aussi vite que possible un concept sur une intuition; de l'autre, de prolonger au maximum l'absence de concept afin de jouir aussi longtemps que faire se peut de ce que l'intuition donne. Il en va de même pour le goût, pour lequel il nous faut distinguer l'utilisation de nos papilles afin d'identifier des objets - comme des douaniers se passant une poudre blanche sur les gencives afin d'identifier, grâce à son fort goût en ammoniaque, de la cocaïne -, de renoncement à toute identification de ce que nous avons dans la bouche dans le seul but d'en jouir et de se laisser emporter par un plat. Il y ainsi deux phénoménalités selon le goût : « le goût peut donner l'intuition d'objets et l'épuiser dans un concept. Pourtant d'une autre part, le goût peut s'exercer sur ce qui échappe à tout concept. » (Marion, 2005a, p. 162). Enfin, il en va de même pour ce qu'il en est de l'ouie comme nous le montre l'exemple de l'opéra. Tout 
comme les autres sens, l'ouïe peut être utilisée dans une phénoménalité objectale, comme lorsque nous nous concentrons pour écouter une conférence. Ici, il s'agit de soumettre ce que nous entendons au concept et à la cohérence des concepts. Mais nous pouvons presque dire qu'obsédés par le sens des sons que nous entendons, nous n'écoutons presque pas la voix qui les prononce. Au contraire, nous pouvons imaginer que l'ouïe soit utilisée selon une phénoménalité saturée et alors, nous n'entendons presque plus le sens des mots prononcés mais jouissons simplement de la voix qui les prononce. Cela est particulièrement clair dans le cas de l'opéra puisque nous pouvons parfaitement apprécier l'opéra sans comprendre l'italien ni l'allemand, et que l'opéra Carmen, de Bizet, est extrêmement écouté et apprécié dans des pays non-francophones ${ }^{29}$. Ainsi, la violence mimétique peut être stoppée si nous ne désirons plus des objets mais portons un nouveau regard sur ce que nous désirons. Cela n'est possible que parce que la saturation n'est pas tant un type de phénomène qu'une modalité de leur réception. Si bien qu'elle ne s'adresse pas à un petit nombre de phénomènes mais peut, en droit, s’appliquer à leur immense majorité.

\section{Conclusion}

IL Y A AINSI DANS LA PHÉNOMÉNOLOGIE de la donation de Marion une sortie possible à la clôture dans laquelle nous plonge la théorie mimétique. Nous avons pu montrer, en effet, que cette fermeture provenait d'un cheminement de l'objet à lêtre totalement porté par une conception visuelle de la construction des objets. Ce faisant, Girard demeure prisonnier de la métaphysique parce que même lorsqu'il envisage une sortie de la violence par l'imitatio Christi, c'est encore en termes d'image et de représentation qu'il pense la relation entre le Christ et le Père, figeant à nouveau le renvoi de l'un à l'autre dans la présence et l'être. La phénoménologie de Marion, pour sa part, nous permet de rompre cette logique grâce à la phénoménalité perçue selon la modalité des phénomènes saturés. Elle nous permet d'opposer à l'image, à la présence et à l'être, le modèle du signe et de l'indifférence à l'être : «à la similitude (et à la rivalité qu'elle provoque) se

29 Nous pourrions même, en poussant cette double phénoménalité à l'extrême, nous demander s'il ne faut pas ne pas comprendre la langue d'un opéra pour pouvoir vraiment l'apprécier pleinement. 
substitue la fidélité (et la communion intentionnelle qu’elle permet). La marque, comme tout signe, ne prétend pas (se) donner à voir, mais entend (se) faire reconnaître comme le prototype qui la marque. » (Marion, 1991, p. 152). Ces phénomènes saturés n'étant pas construits ni constitués par le sujet - et donc à sa mesure - ils échappent à la clôture de la violence mimétique parce que bien plus que de représenter des objets, ils font signe vers un apparaître que nous ne mầtrisons pas en ce qu'il déborde tout concept que nous pouvons en former : « Le phénomène saturé excède en effet les catégories et les principes de l'entendement - [...]. » (Marion, 2005b, p. 57). Ainsi, les phénomènes saturés échappent au jeu de miroir dans lequel nous plonge l'idolâtrie objectale et nous permettent de ne plus entrer dans les jeux de regards en face à face qui font le lit de la violence mimétique. Ils peuvent rompre la boucle autoréférentielle des idoles objectales parce que eux seuls font signe vers un ailleurs qui nous dépasse. Marion permet donc de penser, par sa phénoménologie, une relève du paradigme de l'idole par celui de l'icône. Toutefois, ce modèle de l'icône contre l'idole ne fonctionne quà l'unique condition que nous acceptions la banalité des phénomènes saturés et que nous fassions de la saturation, non pas un type de phénomène, mais un type de réception de tout phénomène : «Tout peut donc devenir un phénomène saturé pour autant que la façon dont il se donne ne se trouve pas rabattue, comme l'impose la quotidienneté du monde technique, sur l'objectivité univoque. » (Marion, 2012, p. 151).

Face à la violence globale qui nous menace aujourd'hui, il ne s'agit donc ni de renoncer au désir, ni de modifier nos modèles, ni même de changer d’objet de désir. La solution que nous permet de penser la phénoménologie de JeanLuc Marion est au contraire de désirer autre chose que des objets, et puisque les objets ne sont pas des types de chose mais que l'objectité est une interprétation possible des phénomènes, nous pouvons continuer à désirer tout ce que nous souhaitons, à condition de ne pas continuer à percevoir le monde à l'aune de l'objectité. Nous pouvons certes être en rivalité pour la possession d'un tableau si nous le percevons en tant qu'objet, c'est-à-dire si nous l'évaluons financièrement, si nous le percevons comme une marque de statut social ou comme refuge financier lors d'une crise boursière. Mais nous ne pouvons pas être en rivalité pour sa possession si nous le percevons comme phénomène esthétique et non plus comme objet. Sa saturation bloque la rivalité mimétique de possession et 
de privation parce que nous ne le maitrisons plus et que ce qu'il nous donne nous dépasse tous. De la même façon, nous pouvons être en conflit pour la possession d'une femme si celle-ci est perçue comme objet de jouissance, comme objet de plaisir, comme objet de fantasme ou comme objet marquant un statut social. Mais sitôt qu'elle n'est plus perçue sur le mode de l'objectité, la rivalité ne peut plus se construire parce que sa façon d'apparaître dans le monde n'est plus déterminée par la somme des regards se posant sur elle. Echappant à la construction de son apparaître par le regard de certains sujets, elle échappe ce faisant à la spirale mimétique de la violence. Ainsi, la phénoménologie de la donation apparaît comme la seule option possible à la sortie de la crise mimétique qui fait rage, aujourd'hui plus que jamais, en nous demandant de changer notre regard, c'est-à-dire notre phénoménalité, et nous poussant à comprendre que la difficulté aujourd'hui n'est pas tant de savoir donner que de savoir recevoir.

Références

Alquié, F. (1950). La découverte métaphysique de l'homme chez Descartes. Paris : Presses Universitaires de France.

Anspach, M. (2002). À charge de revanche, Figures élémentaires de la réciprocité. Paris : Seuil.

Armogathe, J-R. (dir.) (2011). Histoire générale du christianisme. Paris : PUF.

Derrida, J. (1967). De la grammatologie. Paris : Les Éditions de minuit.

Derrida, J. (1980). La carte postale, de Socrate à Freud et au-delà. Paris : Flammarion.

Descartes, R. (1992). Méditations métaphysiques. CEuvres philosophiques tome II (pp. 375-505). Paris : Garnier.

Dupuy, J-P. (2002). Avions-nous oublié le mal ? Penser la politique après le 11 septembre. Paris : Bayard.

Girard, R. (1961). Mensonge romanesque et vérité romantique. Paris : Grasset. Réédition in Girard, R. (2007). De la violence à la divinité. (pp. 29-292) Paris : Grasset.

Girard, R. (1972). La violence et le sacré. Paris : Grasset. Réédition in Girard, R. (2007) De la violence à la divinité (pp. 293-699) Paris : Grasset.

Girard, R. (1999). Je vois Satan tomber comme l'éclair. Paris : Grasset. Réédition (2001), Paris : LGF. 
Girard, R. (2007). Achever Clausewitz. Paris : Carnets Nord. Édition revue et augmentée (2011) Paris : Flammarion.

Heidegger, M. (1986). Etre et temps. Paris : Gallimard.

Kant, E. (1980). Critique de la raison pure. CEuvres Philosophiques I, Despremiers écrits à la Critique de la raison pure (pp. 719-1470), Paris : Gallimard.

Kant, E. (1985). Critique de la faculté de juger. CEuvres Philosophiques II, Des prolégomènes aux écrits de 1791 (pp. 845-1299), Paris : Gallimard.

Levinas, E. (1961). Totalité et infini, Essai sur l'extériorité. La Haye : Martinus Nijhoff.

Levinas, E. (1974). Autrement quêtre ou au-delà de l'essence. La Haye : Martinus Nijhoff.

Levinas, E. (2009). CEuvres 1, Carnets de captivité et autres inédits. Paris: Grasset. Marion, J-L. (1975). Sur l'ontologie grise de Descartes, Science cartésienne et savoir aristotélicien dans les Regulae. Paris : Vrin.

Marion, J-L. (1982). Dieu sans l'être. Paris : Fayard. Réédition (1991, 2002) Paris : PUF.

Marion, J-L. (1991). La croisée du visible. Paris : La Différence. Réédition (1996, 2007) Paris : Presses Universitaires de France.

Marion, J-L. (1997). Étant donné, Essai d'une phénoménologie de la donation. Paris : PUF. Réédition (2005) Paris : PUF.

Marion, J-L. (2001). De surcroît, Études sur les phénomènes saturés. Paris : PUF. Réédition (2010). Paris : PUF.

Marion, J-L. (2005a). La banalité de la saturation, Le visible et le révélé (pp. 143182), Paris : Cerf.

Marion, J-L. (2005b). Le phénomène saturé, Le visible et le révélé (pp. 35-74), Paris : Cerf.

Marion, J-L. (2012). La rigueur des choses, Entretiens avec Dan Arbib. Paris : Flammarion.

Pascal, B. (2000). Pensées retranchées, CEuvres Complètes tome II (pp. 851-868), Paris : Gallimard.

Ramond, C. (2002). Récits et Images du Rêve, Colloque Le rêve, entre science et philosophie, université Paris 1, UFR de philosophie / ED de philosophie, Resp. Christiane Chauviré, Paris, 19-21 septembre 2002. http://perso. orange.fr/charles.ramond/Recits_et_Images_du_Reve.pdf 
Rey, O. (2016). 2uand le monde s'est fait nombre. Paris : Stock.

Serres, M. (2012). Petites poucettes. Paris : Le Pommier.

Spinoza, B. (1966). Lettre LVI à Hugo Boxel, CEuvres IV (pp. 297-300), Paris : Flammarion.

Vinolo, S. (2010). Critique de la raison mimétique - Girard lecteur de Sartre. C. Ramond (Ed.), La théorie mimétique : de l'apprentissage à l'apocalypse (pp. 59-104). Paris : Presses Universitaires de France.

Vinolo, S. (2012). Dieu n'a que faire de l'être, introduction à l'ouvre de Jean-Luc Marion. Paris : Germina.

Vinolo, S. (2013). La majorité contre la foule. Cités, 53, 2013/1, pp. 87-106.

Vinolo, S. (2016). La tentation moderne de Jean-Luc Marion. Dialogue 55, pp. 343-362.

Vinolo, S. (2017). ¿Qué más da? La estética en Jean-Luc Marion. Escritos, Vol. 25, N. 54, pp. 197-220. 